\title{
On the geometry of quantum indistinguishability
}

\author{
A F Reyes-Lega \\ Departamento de Física, Universidad de los Andes, AA 4976, Bogotá D.C., Colombia \\ E-mail: anreyes@uniandes.edu.co
}

\begin{abstract}
An algebraic approach to the study of quantum mechanics on configuration spaces with a finite fundamental group is presented. It uses, in an essential way, the Gelfand-Naimark and Serre-Swan equivalences and thus allows one to represent geometric properties of such systems in algebraic terms. As an application, the problem of quantum indistinguishability is reformulated in the light of the proposed approach. Previous attempts aiming at a proof of the spin-statistics theorem in nonrelativistic quantum mechanics are explicitly recast in the global language inherent to the presented techniques. This leads to a critical discussion of single-valuedness of wave functions for systems of indistinguishable particles. Potential applications of the methods presented in this paper to problems related to quantization, geometric phases and phase transitions in spin systems are proposed.
\end{abstract}

AMS classification scheme numbers: 81Q70, 81S05, 81Q35

Submitted to: J. Phys. A: Math. Gen. 


\section{Introduction}

As is well-known, there are several proofs of the spin-statistics theorem that are valid for quantum fields. The first versions of the theorem, due to Fierz [1] and Pauli [2], date back to the forties and were valid only for free relativistic quantum fields. The developments that eventually led to the establishment of the theorem in the general case have been described in the book by Duck and Sudarshan [3]. The modern proofs of the theorem, in the framework of (axiomatic/algebraic) quantum field theory, are described in the books by Streater and Wightman [4] and by Haag [5]. Although these proofs use relativistic invariance in an essential way, there are other alternative approaches that do not make use of the full Poincaré covariance of the theory. One of them, based on Schwinger's action principle, has been pioneered by Sudarshan [3, 6].

A more radical point of view, that has been explored for several years, is based on the idea that it might be possible to explain the spin-statistics connection from within (nonrelativistic) quantum mechanics. There have been many proposals along this line of thought. One of them, proposed by Balachandran and coworkers [7], uses classical configuration spaces of identical particles and resembles the work of Finkelstein and Rubinstein on kink configurations for nonlinear fields [8]. Even though their approach does not make direct use of quantum field theory, the idea of pair creation/annihilation is incorporated indirectly in the topology of the configuration space. A similar approach has also been developed, independently, by Tscheuchner [9] (see also [10]).

More recently, Berry and Robbins [11] have developed a new approach in which no use of relativity or quantum field theory is made. The basic idea of Berry and Robbins is to construct an operator that implements the simultaneous and continuous exchange of the particles' spin and position labels. An essential part of their approach is the imposition of single-valuedness of the total wave function under exchange, as a way to incorporate indistinguishability without using the symmetrization postulate. Their construction has the virtue of being very explicit and of giving the correct statistics sign, for any value of the spin. Unfortunately (as realized by the same authors) this construction is not the only one meeting their requirements: There are other possibilities, allowing for a violation of the spin-statistics connection [12]. In spite of the fact that it does not provide a "proof" of the spin-statistics theorem, the BerryRobbins approach [11, 12, 13, 14] has received a lot of attention [15, 16, 17, 18] and has inspired new questions and developments, both in mathematics [19, 20, 21] and physics [22, 23, 24, 25, 26, 27].

Among these, a proposal to study the spin-statistics connection using tools from noncommutative geometry was made by Paschke [28]. Using only the $S U(2)$ symmetry of the algebra $C\left(S^{2}\right)$ of continuous functions on the sphere, he proved that the subspace consisting of all odd functions in $C\left(S^{2}\right)$ can be regarded, via the Serre-Swan equivalence, as the space of sections of a line bundle over the projective space $\mathbb{R} P^{2}$. Using this result, we recognized that the exchange-rotation operator of Berry and Robbins could be rewritten as a projector. This projector turned out to be given precisely by direct sums 
of the projectors constructed by Paschke [25]. These results, in turn, led to a critical discussion of single-valuedness and its role for the spin-statistics connection [25, 27]. The discussion, though, was based on results that are valid only for 2 particles. Therefore, the main purpose of the present paper is to develop and generalize the framework on which the discussions in [25] and [27] are based. Although the main motivation for the present paper has been the spin-statistics connection, the results obtained fit in the more general context of quantum mechanics on general configuration spaces. As explained in the last section, there are potential applications of the techniques developed in this paper to the study of certain quantization problems, to problems related to Berry phases and to the study of spin systems.

Before embarking on the technical part of the paper, I want to make some comments regarding the status of what can be called the "configuration space approach" to spinstatistics, in view of the opinion among several authors that any attempt at explaining the connection without relativistic quantum field theory is doomed to fail (cf. [18, 29]).

To a big extent, the interest in alternative proofs of the spin-statistics theorem in the last years increased due to Neuenschwander [30] who, motivated by a -now famousremark of Feynman, asked for a simple explanation of spin-statistics. Some direct responses soon appeared. Currently, numerous attempts at simple proofs of the theorem can be found in the literature. Unfortunately, a significant part of this production is based on assumptions and methods lacking either a clear physical justification or a sound mathematical basis, this leading (in some cases) to rather superficial treatments of the problem. Critiques to such oversimplified approaches can be found, e.g., in Hilborn [31, Romer [32] and Duck and Sudarshan [3, 33].

It seems, therefore, that Feynman's demand for simplicity -in a straightforward sense- cannot be met. But one should also bear in mind that many of the nonstandard approaches have a theme in common, one that touches upon an important part of quantum theory: The study of quantum mechanics on general configuration spaces [34]. This more general problem touches on the very foundations of quantum mechanics while connecting representation theory with geometry, topology and measure theory, as can be seen, for instance, from the work of Mackey [68]. Moreover this general problem has attracted the attention of physicists ever since the early days of quantum theory. For example, the early investigations by Pauli [35, 36] were motivated by the search for a single-valuedness criterion for wave functions. The unavailability of a classical analogue for spin led Bopp and Haag [37] to study the quantum theory of the rotation group, regarded as a configuration space. The crucial contributions made by Schulman [38] and by Laidlaw and DeWitt [39] were based on the observation (due to Schulman) that the path integral in a multiply connected space has to be modified with respect to its usual form. Nowadays it is clear that the topology of the configuration space plays a prominent role in the quantization of a classical system (cf. [34, 40, 41, 42, 43, 44, 45] and references therein). More generally, topological ideas -not necessarily related to quantization- have permeated many branches of physics, including condensed matter physics and quantum field theory. Thus, many of the nonstandard approaches to spin-statistics, rather than 
seeking a simple explanation for the connection, aim at a better understanding of it, stemming (at least partially) from the properties of the configuration space. This idea goes back, at least, to the works of Finkelstein and Rubinstein [8], Laidlaw and DeWitt [39], and Leinaas and Myrheim [42]. Many ideas inspired by those works have been put forward in the last decades. Perhaps the reader would agree that none of them can claim to have led to a substantially deeper understanding of spin-statistics. Why, then, the increasing interest in the topic? Indeed, the vast literature dealing with the spin-statistics connection (and particularly with attempts to find new and simpler ways to understand it) gives a hint as to the feeling of discomfort of many authors regarding its current physical status.

In my opinion, the current interest comes from different sources:

- The need to re-examine the connection between spin and statistics in view of new developments in theoretical physics as, for instance, in the context of higher dimensional theories [46], quantum gravity [47, 48], algebraic quantum field theory [9] or non-commutative quantum field theory [49].

- The fact that many quantum phenomena that depend crucially on the spin-statistics connection take place outside the realm of relativistic quantum field theory leads one to wonder whether there is a possible explanation for it based solely on the principles of quantum mechanics.

- The current experimental search for violations of the exclusion principle: The recent Trieste conference on theoretical and experimental aspects of the spin-statistics connection showed that there is a real interest from both experimentalists and theoreticians in deepening our understanding of the connection and that intense experimental research around the topic is starting to be done [50].

Let us finish this introduction with a description of the rest of the paper. Section 2 contains the proofs of the theorems announced in [25] and [27]. These theorems, along with their proofs, constitute the main contribution of the present paper. Section 3 presents two applications of the results from section 2 to the problem of quantum indistinguishability. Here, our previous analysis of the Berry-Robbins approach is briefly reviewed. In section 4 we present our conclusions and an outlook for future work. In Appendix A we explain, for the benefit of the reader unfamiliar with these tools, the equivalence between projective modules and vector bundles. Finally, Appendix B contains background material on group representations that the reader might find useful in order to understand the motivation behind the constructions presented in section 2 . 


\section{An algebraic characterization of flat bundles on spaces with a finite fundamental group}

Let $\mathcal{Q}$ be a compact manifold with fundamental group $\pi_{1}(\mathcal{Q})$. Let $C(\widetilde{\mathcal{Q}})$ denote the algebra of continuoust, complex functions on $\widetilde{\mathcal{Q}}$, the universal covering space of $\mathcal{Q}$. The (right) action of $\pi_{1}(\mathcal{Q})$ on $\widetilde{\mathcal{Q}}$ induces, in a natural way, a representation of $\pi_{1}(\mathcal{Q})$ on $C(\widetilde{\mathcal{Q}})$. On the other hand, the homeomorphism $\widetilde{\mathcal{Q}} / \pi_{1}(\mathcal{Q}) \cong \mathcal{Q}$ induces, at the level of functions, an algebra isomorphism between $C(\mathcal{Q})$ and $C(\widetilde{\mathcal{Q}})^{\pi_{1}(\mathcal{Q})}$, the latter being the subalgebra of $C(\widetilde{\mathcal{Q}})$ consisting of all $\pi_{1}(\mathcal{Q})$-invariant functions. These facts allow one to regard $C(\widetilde{\mathcal{Q}})$ as a module over $C(\mathcal{Q})$. The main purpose of this section is to obtain an algebraic characterization of the set of flat complex vector bundles over $\mathcal{Q}$, when $\pi_{1}(\mathcal{Q})$ is a finite group, through a detailed study of the module structure of $C(\mathcal{Q})$. The relevance of this characterization for the study of quantum indistinguishability has been discussed at length in references [25, 27]. Let us remark that theorem 2.1, lemma 2.2, lemma 2.3 and theorem 2.10 constitute the original contribution of the present paper, whereas theorems 2.5 and 2.7 are well-known results that have been included for the sake of completeness.

\subsection{Decomposition of $C(\widetilde{\mathcal{Q}})$ into a direct sum of $C(\mathcal{Q})$-submodules}

Let $G$ be a finite group. A well-known result from representation theory states that every irreducible representation of $G$ appears in the regular representation, with a multiplicity equal to its dimension. It is then natural to consider the projection maps that single out each one of these representations. From the explicit form of the projection operators, one can easily conclude that the representation space $\mathcal{F}(G)$ of the regular representation (the vector space of complex valued functions on $G$ ) has a basis consisting of all matrix elements of the irreducible representations of $G$. The generalization of this fact to compact Lie groups is the celebrated Peter-Weyl theorem [53]. In this case, the representation space is $L^{2}(G)$. Something similar occurs when we have an action $l: G \times M \longrightarrow M$ of a group $G$ on a space $M$. Such an action induces, in a natural way, a linear action of $G$ on the space of complex functions on $M$ : If $f$ is such a function and $g \in G$, then $g \cdot f(m):=f\left(l_{g^{-1}}(m)\right)$. In the two cases mentioned above, the space $M$ is the group itself and the action $l$ is given by the group multiplication. But we can also let $M$ be a more general space, on which $G$ acts. If $M$ is a compact manifold and $G$ is a finite group acting freely on $M$, then the quotient space $M / G$ is again a manifold. In this case, the action $l$ also induces a representation of $G$ on the space $C(M)$ of complex continuos functions on $M$. The interesting point is that the decomposition of $C(M)$ into irreducible representations, which is achieved purely by algebraic means, has a geometric interpretation, in the sense that it also induces a decomposition of $C(M)$ into a sum of finitely generated, projective $C(M / G)$-modules. It then follows, from the Serre-Swan

$\ddagger$ Here we will consider spaces of continuous functions (or sections) but in the applications we have in mind, it is the Hilbert space completion with respect to a suitable inner product what we are interested in. 
theorem, that each such subspace of $C(M)$ can be regarded as the space of sections of some vector bundle over $M / G$. Therefore, we shall now seek to establish the following result:

Theorem 2.1. Let $G$ be a finite group acting freely on the left on a compact manifold $M$ by $l: G \times M \rightarrow M$. Then, the decomposition of $C(M)$ into irreducible representations induces, simultaneously, a decomposition of $C(M)$ as a direct sum of finitely generated and projective $C(M / G)$-modules.

We will divide the proof of this theorem in two parts (lemmas 2.2 and 2.3 below). Let $C^{\mathrm{G}}(M)$ be the subspace of $C(M)$ consisting of all $G$-invariant functions:

$$
C^{\mathrm{G}}(M):=\left\{f \in C(M) \mid f\left(l_{g}(x)\right)=f(x) \forall x \in M\right\} .
$$

Since the product of two invariant functions is again an invariant function, this subspace is also an algebra (and, in particular, a ring). We can, therefore, regard $C(M)$ as a module over $C^{\mathrm{G}}(M)$. Moreover, since $C^{\mathrm{G}}(M)$ and $C(M / G)$ are isomorphic algebras, we can also regard $C(M)$ as a $C(M / G)$-module. Let now $\widehat{G}$ denote the set of equivalence classes of irreducible representations of $G$. We will tacitly identify each class $[R]$ in $\widehat{G}$ with a concrete representative $R \in[R]$, chosen to be also a unitary representation. The dimension of the representation $R$ will be denoted by $n_{R}$. Define now, for each $R \in \widehat{G}$ and for each pair of indices $i, j \in\left\{1, \ldots, n_{R}\right\}$, a map $E_{i j}^{R}: C(M) \rightarrow C(M)$, as follows:

$$
E_{i j}^{R} f(x):=\frac{n_{R}}{|G|} \sum_{g \in G} R_{i j}\left(g^{-1}\right) f\left(l_{g^{-1}}(x)\right), \quad(f \in C(M), x \in M) .
$$

Note the obvious similarity between this definition and the one given in (B.12). Functions in the image of each $E_{i j}^{R}$ (for $R$ fixed) are related through the action of $G$ on $C(M)$. Indeed, for $f \in C(M)$ and $g \in G$, we have:

$$
\begin{aligned}
& g \cdot\left(E_{i j}^{R} f\right)=\sum_{k=1}^{n_{R}} R_{k j}(g)\left(E_{i k}^{R} f\right), \\
& E_{i j}^{R}(g \cdot f)=\sum_{k=1}^{n_{R}} R_{i k}(g)\left(E_{k j}^{R} f\right) .
\end{aligned}
$$

The maps $E_{i j}^{R}$ obey, furthermore, the following important orthogonality relations:

$$
E_{i k}^{R} E_{m n}^{R^{\prime}}=\delta_{R, R^{\prime}} \delta_{i, n} E_{m k}^{R} .
$$

This follows directly from the orthogonality relations for the matrix elements of the representations. Taking $R=R^{\prime}$ and $i=k=m=n$ in (4), we see that the operator $E_{i i}^{R}$ is a projection operator. Moreover, summing over all $R$ in $\widehat{G}$ and (for each $R$ ) over all $i$, we obtain, as in (B.11), a completeness relation $(f \in C(M))$ :

$$
f=\sum_{R \in \widehat{G}} \sum_{i=1}^{n_{R}} E_{i i}^{R} f .
$$


In fact, we have:

$$
\begin{aligned}
\sum_{R \in \widehat{G}} \sum_{i=1}^{n_{R}} E_{i i}^{R} f(x) & =\sum_{R \in \widehat{G}} \sum_{i=1}^{n_{R}}\left[\frac{n_{R}}{|G|} \sum_{g \in G} R_{i i}\left(g^{-1}\right) f\left(l_{g^{-1}}(x)\right)\right] \\
& =\sum_{R \in \widehat{G}} \sum_{g \in G} \frac{n_{R}}{|G|} \chi^{R}\left(g^{-1}\right) f\left(l_{g^{-1}} x\right) \\
& =\sum_{g \in G} \frac{1}{|G|} \underbrace{\sum_{R \in \widehat{G}} n_{R} \chi^{R}\left(g^{-1}\right)}_{=|G| \delta_{e, g}} f\left(l_{g^{-1}} x\right) \\
& =f(x) .
\end{aligned}
$$

Lemma 2.2. Let $R \in \widehat{G}$ and $i \in\left\{1, \ldots, n_{R}\right\}$ and define $\mathcal{E}_{i}^{R}:=E_{i i}^{R}(C(M))$. Then $\mathcal{E}_{i}^{R}$ is a finitely generated $C(M / G)$-module. Moreover, $C(M)$ can be written, as a $C(M / G)$ module, as the direct sum

$$
C(M)=\bigoplus_{R \in \widehat{G}} \bigoplus_{i=1}^{n_{R}} \mathcal{E}_{i}^{R}
$$

Proof. Let $q: M \rightarrow M / G$ denote the quotient map induced by the (smooth) action $l: G \times M \rightarrow M$. Since $G$ is finite and the action $l$ is free, it follows that $l$ is properly discontinuous without fixed points, so the standard theorem [54] for quotients of such actions applies, showing that $M / G$ is also a smooth manifold. Following the proof of that theorem, one sees that it is possible to choose charts for $M$ of the form $\left\{\left(\tilde{U}_{\alpha, g}, \tilde{\varphi}_{\alpha, g}\right)\right\}_{\alpha \in I, g \in G}$, with $I$ finite ( $M$ is assumed to be compact) and with the following properties:

For each $\alpha$ in $I, \tilde{U}_{\alpha, g}=g \cdot\left(\tilde{U}_{\alpha, e}\right)$ for all $g \in G$.

For each $\alpha$ in $I, \tilde{U}_{\alpha, g} \cap \tilde{U}_{\alpha, g^{\prime}}=\emptyset$ whenever $g \neq g^{\prime} ; g, g^{\prime} \in G$.

Here, the notation $l_{g}(x)=g \cdot x$ for the action has been used. It follows immediately from these properties that, for a given $\alpha, q\left(\tilde{U}_{\alpha, g}\right)=q\left(\tilde{U}_{\alpha, e}\right)$ holds, for all $g$. Put now $U_{\alpha}:=q\left(\tilde{U}_{\alpha, g}\right)$, with $g \in G$ arbitrarily chosen. The collection $\left\{U_{\alpha}\right\}_{\alpha \in I}$ gives an open cover for $M / G$, with the following property:

$$
q^{-1}\left(U_{\alpha}\right)=\bigcup_{g \in G} \tilde{U}_{\alpha, g}=\bigcup_{g \in G} g \cdot\left(\tilde{U}_{\alpha, e}\right),
$$

i.e. the inverse image of $U_{\alpha}$ is a union of pairwise disjoint neighborhoods. Let now $\left\{\phi_{\alpha}\right\}_{\alpha \in I}$ be a partition of unity for $M / G$, subordinated to the cover $\left\{U_{\alpha}\right\}_{\alpha \in I}$ ( $\operatorname{Supp} \phi_{\alpha} \subset U_{\alpha} \subset M / G$ and with the convention that $\sum_{\alpha \in I} \phi_{\alpha}^{2}=1$ ).

Each $\phi_{\alpha}$ gives place to a pull-back function $q^{*} \phi_{\alpha} \in C^{\infty}(M)$, defined through $q^{*} \phi_{\alpha}:=\phi_{\alpha} \circ q$. This, in turn, can be used to define, for each $\alpha$, the following map:

$$
\psi_{\alpha}(x):=\left\{\begin{array}{cc}
q^{*} \phi_{\alpha}(x), & \text { if } x \in \tilde{U}_{\alpha, e} \\
0, & \text { otherwise. }
\end{array}\right.
$$


Notice that these are indeed smooth functions, because of ( $8 b$ ) and the fact that $\operatorname{Supp}\left(q^{*} \phi_{\alpha}\right)=q^{-1}\left(\operatorname{Supp} \phi_{\alpha}\right) \subseteq \bigcup_{g \in G} \tilde{U}_{\alpha, g}$. More generally, let us define, for each $f \in C(M)$, the following (continuous) functions $(\alpha \in I, g \in G)$ :

$$
f_{\alpha, g}(x):=\left\{\begin{array}{cl}
\left(\left(q^{*} \phi_{\alpha}\right) f\right)(x), & \text { if } x \in \tilde{U}_{\alpha, g} \\
0, & \text { otherwise. }
\end{array}\right.
$$

Define now, for $f \in C(M)$, the invariant functions $(x \in M)$

$$
f_{\alpha, g}^{\mathrm{s}}(x):=\sum_{h \in G} f_{\alpha, g}\left(h^{-1} \cdot x\right) .
$$

From these definitions we immediately obtain the following identity:

$$
\sum_{g \in G} f_{\alpha, g}^{\mathrm{s}}(x) \psi_{\alpha}\left(g^{-1} \cdot x\right)=\phi_{\alpha}([x])^{2} f(x) .
$$

Summing over $\alpha$, we then get

$$
f(x)=\sum_{\alpha \in I} \phi_{\alpha}([x])^{2} f(x)=\sum_{\alpha \in I} \sum_{g \in G} f_{\alpha, g}^{\mathrm{s}}(x) \psi_{\alpha}\left(g^{-1} \cdot x\right) .
$$

Assume now that $f \in \mathcal{E}_{i}^{R}$, i.e. that $f=E_{i i}^{R} f$. Then, using (21), (14) and (하), we obtain:

$$
\begin{aligned}
f(x) & =E_{i i}^{R} f(x)=\left[E_{i i}^{R}\left(\sum_{\alpha \in I} \sum_{g \in G} f_{\alpha, g}^{\mathrm{s}} g \cdot \psi_{\alpha}\right)\right](x) \\
& =\left[\sum_{\alpha \in I} \sum_{g \in G} E_{i i}^{R}\left(f_{\alpha, g}^{\mathrm{s}} g \cdot \psi_{\alpha}\right)\right](x) \\
& =\sum_{\alpha \in I} \sum_{g \in G} f_{\alpha, g}^{\mathrm{s}}(x)\left[E_{i i}^{R}\left(g \cdot \psi_{\alpha}\right)\right](x) \\
& =\sum_{\alpha \in I} \sum_{g \in G} f_{\alpha, g}^{\mathrm{s}}(x) \sum_{k=1}^{n_{R}} R_{i k}(g)\left[E_{k i}^{R} \psi_{\alpha}\right](x) \\
& =\sum_{\alpha \in I} \sum_{k=1}^{n_{R}}\left(\sum_{g \in G} f_{\alpha, g}^{\mathrm{s}}(x) R_{i k}(g)\right)\left[E_{k i}^{R} \psi_{\alpha}\right](x) .
\end{aligned}
$$

Define now $\psi_{\alpha, i k}^{R}:=E_{k i}^{R} \psi_{\alpha}$ (the reason for inverting the order of the indices will become clear below) and observe that

- $f_{\alpha, g}^{\mathrm{s}}$ is invariant under $G$. Therefore, $\sum_{g \in G} f_{\alpha, g}^{\mathrm{s}} R_{i k}(g) \in C^{G}(M) \cong C(M / G)$.

- For each $\alpha \in I$ and each $k \in\left\{1, \ldots, n_{R}\right\}$, the function $\psi_{\alpha, i k}^{R}$ belongs to $\mathcal{E}_{i}^{R}$. This follows directly from (41).

We have therefore proved that $\mathcal{E}_{i}^{R}$ is a finitely generated $C(M / G)$-module since, as (15) shows, $\left\{\psi_{\alpha, i k}^{R}\right\}_{\alpha \in I, 1 \leq k \leq n_{R}}$ constitutes a finite set of generators for $\mathcal{E}_{i}^{R}$ as a module over $C^{G}(M) \cong C(M / G)$. The fact that $C(M)=\bigoplus_{R \in \widehat{G}} \bigoplus_{i=1}^{n_{R}} \mathcal{E}_{i}^{R}$ follows directly from (5).

Lemma 2.3. There is, for every $R \in \widehat{G}$, an integer $N_{R}$ and a projector $P^{R}$ such that $P^{R}\left(C(M / G)^{N_{R}}\right) \cong \mathcal{E}_{i}^{R}$ for every $i \in 1, \ldots, n_{R}$. 
Proof. Keeping the same conventions and notation as in the previous lemma, set $N_{R}=n_{R}|I|$ and define a linear map $P^{R}: C(M)^{N_{R}} \rightarrow C(M)^{N_{R}}$ as follows. The free module $C(M)^{N_{R}}$ is a direct sum of $N_{R}$ copies of $C(M)$. Therefore, every element $F$ of $C(M)^{N_{R}}$ can be written as an $N_{R}$-tuple $F \equiv\left(F_{\alpha, j}\right)$, with $\alpha \in I$ and $1 \leq j \leq n_{R}$. Define $P^{R} F$ as the $N_{R}$-tuple whose $(\alpha, j)$ component is given by

$$
\left(P^{R} F\right)_{\alpha, j}:=\frac{|G|^{2}}{\left(n_{R}\right)^{2}} \sum_{\beta \in I} \sum_{k, l=1}^{n_{R}} \overline{\psi_{\alpha, l j}^{R}(x)} \psi_{\beta, l k}^{R}(x) F_{\beta, k}(x),
$$

with the bar denoting complex conjugation. It is useful to regard $P^{R}$ as a $C(M)$-valued $N_{R} \times N_{R}$ (block) matrix. Doing so, one sees that the component $(j, k)$ corresponding to the block $(\alpha, \beta)$ must be given by

$$
\left(P_{\alpha \beta}^{R}(x)\right)_{j k}=\frac{|G|^{2}}{\left(n_{R}\right)^{2}} \sum_{l=1}^{n_{R}} \overline{\psi_{\alpha, l j}^{R}(x)} \psi_{\beta, l k}^{R}(x) .
$$

A straightforward calculation shows that $\left(P^{R}\right)^{2}=P^{R}$. This means that $P^{R}\left(C(M)^{N_{R}}\right)$ is a projective module over $C(M)$. But it turns out that, for any $g \in G$, we have $\left(P_{\alpha \beta}^{R}(g \cdot x)\right)_{j k}=\left(P_{\alpha \beta}^{R}(x)\right)_{j k}$. Therefore, we can use the isomorphism $C^{G}(M) \cong C(M / G)$ in order to obtain a projective module over $C(M / G)$, by letting $P^{R}$ act on $C(M / G)^{N_{R}}$. As shown below, this module is isomorphic to $\mathcal{E}_{i}^{R}$. First let us remark that the module $P^{R}\left(C(M / G)^{N_{R}}\right)$ is generated by the columns of $P^{R}$, considered as $C(M / G)$-valued vectors. Let us now map the column of $P^{R}$ that is labeled by the indices $(\alpha, k)$ to the generator $\psi_{\alpha, i k}^{R}$ of $\mathcal{E}_{i}^{R}$. Since both modules are projective, it is not enough to give a bijection between the sets of generators to obtain a module isomorphism: Both sets of generators must satisfy the same relations. The relations satisfied by the generators of $P^{R}\left(C(M / G)^{N_{R}}\right)$ are obtained from the condition $\left(P^{R}\right)^{2}=P^{R}$. On the other hand, using (4) we obtain the following identity:

$$
\sum_{\alpha \in I} \sum_{l=1}^{n_{R}} \psi_{\alpha, k^{\prime} l}^{R} \overline{\psi_{\alpha, k l}^{R}}=\frac{n_{R}^{2}}{|G|^{2}} \delta_{k^{\prime}, k} .
$$

Using this identity, together with (16), we get:

$$
\sum_{\alpha \in I} \sum_{k=1}^{n_{R}}\left(P_{\alpha \beta}^{R}([x])\right)_{k j} \overline{\psi_{\alpha, i k}^{R}(x)}=\overline{\psi_{\beta, i j}^{R}(x)} .
$$

This means that the generators of $\mathcal{E}_{i}^{R}$ satisfy the same relations as the columns of $P^{R}$. Therefore, the $C(M / G)$-linear extension of the map used to identify the generators gives the desired isomorphism.

Let us make a few remarks about the meaning of this result. Consider a principal bundle $(G, P, M)$ with total space $P$, structure group $G$ and base space $M$. Let $\xi=P \times_{G} V$ denote a vector bundle with fibre $V$, associated to $P$ through some representation of $G$ on $V$. There is a well known theorem according to which every section $s$ of $\xi$ can represented by a vector valued function $\chi_{s}: P \rightarrow V$ which is equivariant with respect to the $G$-actions on $P$ and $V$. Conversely, every $G$-equivariant 
map from $P$ to $V$ represents some section of the bundle $\xi$. The result we have obtained is similar to that theorem in the sense that the triple $(G, M, M / G)$ can be regarded as a principal bundle. Sections of a vector bundle associated to this principal bundle through a representation $R$ of $G$ on a vector space $V$ can therefore be regarded as equivariant maps from $M$ to $V$. If $\operatorname{dim} V>1$, these maps will necessarily be vector valued. What theorem 2.1 states is that these same sections can in fact be represented by scalar (i.e. complex valued) functions on $M$. At first sight, it might appear as counterintuitive to claim that a section of a bundle of rank higher than one over $M / G$ can be represented by a scalar function on $M$, but as the proof of the theorem shows, this is indeed the case. According to a theorem of Milnor [55], every flat vector bundle over $M / G$ is associated to the principal bundle $(G, M, M / G)$ through some representation of $G$. From this point of view, theorem 2.1 says that every section of any flat vector bundle over $M / G$ can be represented by a complex function on $M$. The fact that these bundles are flat is reflected in the fact that the connections become, basically, the exterior derivative on $M$. From a practical point of view, this could be a very convenient way of working with such flat bundles. Potential applications of these techniques to quantization problems, Berry phases and spin systems will be discussed in the last section.

\subsection{Invariant sections}

Pull-backs of vector bundles can be neatly expressed in terms of tensor products between modules of sections and algebras of functions [56. This algebraic description takes a particularly interesting form when applied to pull-backs of quotients by finite groups, a form that turns out to be essential for discussions of quantum indistinguishability [25, 27]. Let us start this section recalling some basic mathematical facts.

Definition 2.4. A vector bundle $\xi=(E(\xi), \pi, M)$ over the $G$-space $M$ is called a $G$-bundle when the following conditions hold:

- The total space $E(\xi)$ is itself a $G$-space (the corresponding action being denoted with $\tau$ ).

- The projection $\pi$ is $G$-equivariant, i.e. $\pi \circ \tau_{g}=\rho_{g} \circ \pi$ for all $g$ in $G$.

- The restriction $\left.\tau_{g}\right|_{\pi^{-1}(m)}: \pi^{-1}(m) \longrightarrow \pi^{-1}(g \cdot m)$ of the action to the fibers is a vector space isomorphism.

A morphism between two $G$-bundles is a $G$-equivariant bundle morphism. The notation $\xi_{1} \cong_{G} \xi_{2}$ will be used whenever $\xi_{1}$ and $\xi_{2}$ are equivalent as $G$-bundles.

For $G$ finite, we have:

Theorem 2.5 (cf.[57]). If $M$ is $G$-free, there is a bijective correspondence between $G$ bundles over $M$ and bundles over $M / G$ by $\eta \rightarrow \eta / G$. 
The precise meaning of this theorem is the following. Let $q: M \rightarrow M / G$ denote the canonical projection and let $\xi$ be a vector bundle over $M / G$. Then, the pull-back $q^{*}$ induces -in a natural way- an action $\tau_{\xi}$ of $G$ on $E\left(q^{*} \xi\right)$, given by

$$
\tau_{\xi}(g,(m, y))=g \cdot(m, y):=(g \cdot m, y), \quad g \in G, \quad(m, y) \in E\left(q^{*} \xi\right)
$$

This action is also free, implying that the quotient $E\left(q^{*} \xi\right) / G$ is also a manifold. One then shows that $E\left(q^{*} \xi\right) / G$ is the total space of a vector bundle (denoted $q^{*} \xi / G$ ) over $M / G$, which is isomorphic to $\xi: q^{*} \xi / G \cong \xi$. On the other hand, let $\eta$ be a $G$-bundle over $M$. The quotient $E(\eta) / G$ is -again- the total space of a bundle, but this time over $M / G$. Its pull-back turns out to be $G$-isomorphic to $\eta: q^{*}(\eta / G) \cong_{G} \eta$.

Consider now a continuous map $\phi: M \rightarrow N$ and a vector bundle $\xi$ over $N$. Noticing that $\phi$ induces a ring homomorphism $\phi^{*}: C(N) \rightarrow C(M)$ (through $\left.f \mapsto \phi^{*} f:=f \circ \phi\right)$, we obtain a $C(N)$-module structure on $\Gamma\left(\phi^{*} \xi\right)$ :

$$
\begin{aligned}
C(N) \times \Gamma\left(\phi^{*} \xi\right) & \longrightarrow \Gamma\left(\phi^{*} \xi\right) \\
(f, s) & \longmapsto f \cdot s:=\left(\phi^{*} f\right) s .
\end{aligned}
$$

If for any given section $\sigma \in \Gamma(\xi)$ we define a new one by

$$
\begin{aligned}
\phi^{*} \sigma: M & \longrightarrow E\left(\phi^{*} \xi\right) \\
x & \longmapsto(x, \sigma \circ \phi(x)),
\end{aligned}
$$

then we obtain the following homomorphism of $C(N)$-modules:

$$
\begin{aligned}
F^{\phi}: \Gamma(\xi) & \longrightarrow \Gamma\left(\phi^{*} \xi\right) \\
\sigma & \longmapsto F^{\phi}(\sigma) \equiv \phi^{*} \sigma .
\end{aligned}
$$

$F^{\phi}$ is clearly a $C(N)$-linear map:

$$
F^{\phi}(f \cdot \sigma)=\phi^{*}(f \cdot \sigma) \stackrel{\text { 22 }}{=}(f \circ \phi) \phi^{*} \sigma \stackrel{21]}{=} f \cdot \phi^{*} \sigma=f \cdot F^{\phi}(\sigma) .
$$

Remark 2.6. It is important to remark that $F^{\phi}$ is not, in general, an isomorphism. Indeed, although we may choose generators for $\Gamma\left(\phi^{*} \xi\right)$ of the form $\sigma_{i}^{\prime}=F^{\phi}\left(\sigma_{i}\right)$, we see from $\operatorname{Im}\left(F^{\phi}\right)=\left\{\sum_{i}\left(f_{i} \circ \phi\right) \sigma_{i}^{\prime} \mid f_{i} \in C(N)\right\}$ that $F^{\phi}$ is not surjective in general, because the elements in the image module are only linear combinations of the generators over the subspace $\phi^{*}(C(N))$ of $C(M)$. In other words: In order to remain inside $\operatorname{Im}\left(F^{\phi}\right)$, the generators $\sigma_{i}^{\prime}$ may be multiplied only by elements of $C(N)$.

The previous remark suggests that we might obtain $\Gamma\left(\phi^{*} \xi\right)$ from $\operatorname{Im}\left(F^{\phi}\right)$ if we are able to replace, somehow, $C(N)$ by $C(M)$. This change of ring can in fact be performed, with the help of the tensor product, because both $C(M)$ and $\Gamma(\xi)$ can be regarded as $C(N)$ modules. One can therefore define the following homomorphism of $C(M)$-modules:

$$
\begin{aligned}
\Phi: C(M) \otimes_{C(N)} \Gamma(\xi) & \longrightarrow \Gamma\left(\phi^{*} \xi\right) \\
\sum_{k} a_{k} \otimes \sigma_{k} & \longmapsto \sum_{k} a_{k} F^{\phi}\left(\sigma_{k}\right) .
\end{aligned}
$$

This is the key idea behind the following (well-known) result. 
Theorem 2.7 (cf. [56, 58]). The map defined through (24) provides an isomorphism $C(M) \otimes_{C(N)} \Gamma(\xi) \cong \Gamma\left(\phi^{*} \xi\right)$ of $C(M)$-modules.

If -for the situation considered in the previous paragraphs- we set $N \equiv M / G$ and $\phi \equiv q: M \rightarrow M / G$ in theorem 2.7, then we can construct an injective $C(M / G)$-module homomorphism $\Phi^{G}: \Gamma(\xi) \hookrightarrow \Gamma\left(q^{*} \xi\right)$, as follows. The decomposition (7) of $C(M)$ into $C(M / G)$-submodules allows us to write $C(M)$ in the form $C(M)=C^{G}(M) \oplus \mathcal{E}$, where as already remarked $C^{G}(M) \cong C(M / G)$ holds, and with $\mathcal{E}$ having the structure of a projective $C(M / G)$-module. Hence we obtain:

$$
C(M) \otimes_{C(M / G)} \Gamma(\xi) \cong \Gamma(\xi) \oplus\left(\mathcal{E} \otimes_{C(M / G)} \Gamma(\xi)\right) .
$$

Denote with $i: \Gamma(\xi) \hookrightarrow C(M) \otimes_{C(M / G)} \Gamma(\xi)$ the inclusion induced by (25). Making use of theorem 2.7 we can define $\Phi^{G}:=\Phi \circ i$ and in that way obtain the desired result.

Remark 2.8. It is important to notice that, although $\Phi^{G}(\Gamma(\xi))$ and $\Gamma(\xi)$ are isomorphic as $C(M / G)$-modules, $\Phi^{G}(\Gamma(\xi))$ is actually contained in $\Gamma\left(q^{*} \xi\right)$. This means that, although every section from $\Gamma(\xi)$ can be "replaced" by one from $\Phi^{G}(\Gamma(\xi))$, sections from $\Phi^{G}(\Gamma(\xi))$ may only be multiplied by functions in $C^{G}(M)$, if we want to identify $\Phi^{G}(\Gamma(\xi))$ and $\Gamma(\xi)$ as modules.

Remark 2.9. From (24) and (25) we see that $\Phi^{G}(\sigma)=F^{q}(\sigma)$.

It is possible to give a more explicit description of the image of $\Phi^{G}$. In fact, one finds that $\Phi^{G}(\Gamma(\xi))$ equals the space of invariant sections of the pull-back bundle. The next result is very important in connection to our discussion of the single-valuedness condition of Berry and Robbins (cf. [25, 27]).

Theorem 2.10. $\Gamma(\xi) \cong \Phi^{G}(\Gamma(\xi))=\Gamma^{G}\left(q^{*} \xi\right):=\left\{s \in \Gamma\left(q^{*} \xi\right) \mid g \cdot s=s \forall g \in \Gamma\right\}$.

Proof. The first equality is clear, since $\Phi^{G}$ is an injective homomorphism. Every section in $\Phi^{G}(\Gamma(\xi))$ is of the form $q^{*} \sigma$, with $\sigma \in \Gamma(\xi)$. From the definition of $q^{*} \sigma$ (see Eq. (22)) and from the form of the action $\tau$ induced induced on $q^{*} \xi$ by $q$ (see Eq.(20) ) it follows that a section of the form $q^{*} \sigma$ is invariant:

$$
\begin{aligned}
\left(g \cdot q^{*} \sigma\right)(x) & =\tau_{g}\left(g^{-1} \cdot x, \sigma \circ q\left(g^{-1} \cdot x\right)\right) \\
& \stackrel{20}{=}\left(x, \sigma \circ q\left(g^{-1} \cdot x\right)\right) \\
& =(x, \sigma \circ q(x)) \\
& =q^{*} \sigma(x) .
\end{aligned}
$$

Conversely, every invariant section must be of the form $q^{*} \sigma$ : Given $s \in \Gamma\left(q^{*} \xi\right)$, there is a continuous map $y: M \rightarrow E(\xi)$ with $\pi \circ y=q$ and $s: x \mapsto(x, y(x))$. If $s$ is invariant, then $y(g \cdot x)=y(x)$ holds for all $g$ in $G$, so that one can define a section $\sigma \in \Gamma(\xi)$ through $\sigma([x]):=y(x)$, for which $s=q^{*} \sigma$ holds.

Remark 2.11. At this point it might not be very clear what kind of advantage can we obtain from describing all these geometric objects in terms of algebraic ones. In the end, as long as we stay within classical geometry, the theorems of Gelfand-Naimark and 
of Serre-Swan assert that there a complete equivalence, at the level of categories. There are two main reasons for insisting in this approach: (i) at some point one would like to make contact with the (more algebraic) language of quantum field theory, in order to try to interpret the spin-statistics theorem with the geometry of the configuration space (whether this is possible or not is a different issue) and (ii) we have already had the experience, working on a geometric interpretation of the Schwinger construction of Berry-Robbins, that the algebraic approach is really worth pursuing: In that case, the whole intricacy of the Schwinger construction reduces to the assertion that the spin basis is represented by the $C\left(\mathbb{R} P^{2}\right)$-projective module $A_{+} \oplus A_{-}^{3}$, where $A \pm$ stands for the space of continuous even/odd functions over the 2-sphere. As shown in [28, 25], this can be obtained from general symmetry considerations.

\section{Applications to quantum indistinguishability}

The results presented in the previous section were originally motivated by our efforts to understand the Berry-Robbins construction from a geometric perspective [25]. The approach we have chosen (fully exploiting the Serre-Swan equivalence) turns out to be very well suited for the study of several problems in quantum mechanics where multiplyconnected configuration (or parameter) spaces are relevant. We will comment on various such possibilities in section 4 .

Here we will discuss an application to the problem of quantum indistinguishability. It goes directly to the core of the Berry-Robbins approach to spin-statistics. Since this involves the use of the concept of single-valuedness of wave functions, it is convenient to add some comments on it. Let us define

$$
\tilde{Q}_{N}:=\left(\mathbb{R}^{3} \times \cdots \times \mathbb{R}^{3} \backslash \Delta\right),
$$

where $\Delta$ denotes the set of configurations with two or more coinciding particles. According to Leinaas and Myrheim [42], if we want to take into account the intrinsic quantum indistinguishability of the particles but still want to work with wave functions defined over a classical configuration space, we have to consider the quotient space $Q_{N}:=\tilde{Q}_{N} / S_{N}$ (obtained through identification of all permuted configurations) as the physical configuration space. This space is multiply connected, which implies there will be inequivalent quantizations of the same classical system. Each possible quantization of the system will be given by a Hilbert space that is to be obtained as the completion of a space of sections of a certain vector bundle over $Q_{N}$. The elements of that Hilbert space are representatives of rays in the projective Hilbert space and it is important to remark that we are free to multiply any representative by an arbitrary phase factor, without changing the "single-valued" character of the section chosen as representative. In the present context of quantum indistinguishability, the term "single-valued wave function" refers to a section of that bundle. As we have seen in the previous section, it is possible to describe a section on a certain bundle over $Q_{N}$ as a function on the covering space $\tilde{Q}_{N}$. This function will in general acquire different values at points that physically 
correspond to the same configurations of particles. For this reason such functions are sometimes called "multiple-valued". In any case, it should be clear that in all cases relevant to our discussion, including references [11, 42], it is $Q_{N}$ and not $\tilde{Q}_{N}$ the space that is to be considered the physical configuration space.

The main purpose of this section is to illustrate the results of section 2 by means of an application of direct physical interest. The main assertion, questioning the applicability of the single-valuedness condition in the form advocated by Berry and Robbins, might appear as something difficult to accept. Therefore we urge the interested reader to carefully follow the argumentation and explicit computations presented in references [25] and [27] both of which are based on the mathematical tools developed in section 2 .

Very roughly, the strategy of Berry and Robbins in their approach to quantum indistinguishability consists in replacing the standard spin states by position-dependent ones, in such a way that an exchange of position leads, at the same time, to an exchange in the spin degrees of freedom. Thus, instead of working with spin states (in this section I will only be concerned with the 2 particle case) of the form $\left|m, m^{\prime}\right\rangle$, they work with spin states of the form $\left|m, m^{\prime}(\boldsymbol{r})\right\rangle$, where $\boldsymbol{r}$ stands for the relative position vector of the two particles. These transported spin states are required to satisfy a relation of the form $\left|m^{\prime}, m(-\boldsymbol{r})\right\rangle=(-1)^{K}\left|m, m^{\prime}(\boldsymbol{r})\right\rangle$ and also to depend smoothly on $\boldsymbol{r}$ and to satisfy a certain property related to parallel transport. It should be remarked that the existence of such a basis is not at all obvious. The construction presented by Berry and Robbins in [11] makes use of Schwinger's representation of spin and has the virtue of providing a transported spin basis that works for any value of the spin, as well as the physically correct statistics $(K=2 S)$. However, as already remarked, there are other possible constructions satisfying all the requirements but giving the wrong statistics. On the other hand, a construction of a spin basis satisfying their three requirements of smoothness, spin exchange and parallel transport in the case of arbitrary $N$ turns out to be very difficult to implement. This difficulty led the authors of [11] to a mathematical problem that was later on reformulated and solved by Atiyah and collaborators [19, 20, 21]. Harrison and Robbins [24] used these results in order to study generalizations of the Schwinger construction for the case of general $N$.

Returning to the 2 particle case, in the Berry-Robbins approach, wave functions are given by expressions of the form

$$
|\Psi(\boldsymbol{r})\rangle=\sum_{m, m^{\prime}} \psi_{m, m^{\prime}}(\boldsymbol{r})\left|m, m^{\prime}(\boldsymbol{r})\right\rangle,
$$

and are required to satisfy the following single-valuedness condition:

$$
|\Psi(\boldsymbol{r})\rangle=|\Psi(-\boldsymbol{r})\rangle .
$$

The purpose of this condition is to incorporate indistinguishability already at the level of configuration space since, for indistinguishable particles, the points $\boldsymbol{r}$ and $-\boldsymbol{r}$ represent exactly the same configuration. In the case of two particles one can therefore say that 
the physical configuration space is the projective plane $\mathbb{R} P^{2}$, obtained from the twosphere $S^{2}$ through the identification $\boldsymbol{r} \sim \boldsymbol{- r}$. Although the physical motivation for the imposition of the single-valuedness requirement is very clear, its implementation in the specific form (27) is a very subtle matter. To bring it to the point, and in order to illustrate the usefulness of the techniques presented in the previous section, let us highlight the following point.

Remark 3.1. The wave function $|\Psi(\boldsymbol{r})\rangle$ considered by Berry and Robbins is a map $|\Psi(\cdot)\rangle: S^{2} \rightarrow \mathbb{C}^{k}$, from the sphere to a vector space $\mathbb{C}^{k}$, with the value of $k$ depending on the explicit construction of the transported spin basis. However, for a generic configuration space, the wave function will be a section of some vector bundle over the configuration space. Since in the present case the physical configuration space is the projective space $\mathbb{R} P^{2}$, the wave function should be given by a section of some vector bundle over $\mathbb{R} P^{2}$, not over the sphere. For this reason, the wave function $|\Psi(\boldsymbol{r})\rangle$ of Berry and Robbins and, in particular, the single-valuedness condition, must be treated with caution.

Taking this remark into account, let us consider the situation of theorem 2.10, with $M=S^{2}$ and $G=\mathbb{Z}_{2}$. In that case we have $q: S^{2} \rightarrow S^{2} / \mathbb{Z}_{2} \cong \mathbb{R} P^{2}$. Now, let us assume, according to the remark, that the wave function $\varphi$ is a section on a vector bundle $\xi$ over $\mathbb{R} P^{2}$, i.e., $\varphi \in \Gamma(\xi)$. By means of the map $\Phi^{\mathbb{Z}_{2}}$ from theorem 2.10 we then obtain an isomorphic copy of $\varphi$ which is a section of the vector bundle $q^{*} \xi$ (a bundle over $S^{2}$ ). According to theorem 2.10, this new section has well-defined transformation properties under permutations of its argument: It must be invariant with respect to the natural $\mathbb{Z}_{2}$ action on $q^{*} \xi$ (cf. (20) ). In order to obtain an explicit form of this invariance condition, it is convenient to regard $q^{*} \xi$ as a sub-bundle of a trivial vector bundle $S^{2} \times \mathbb{C}^{k}$, for a suitably chosen $k$ (this is always possible). After doing this, we find that there must be a map $|\bar{\varphi}(\cdot)\rangle: S^{2} \rightarrow \mathbb{C}^{k}$ such that $\Phi^{\mathbb{Z}_{2}}(\varphi)(\boldsymbol{r})=(\boldsymbol{r},|\bar{\varphi}(\boldsymbol{r})\rangle)$. Therefore, if we want to keep the interpretation that (because of indistinguishability) the configuration space is $\mathbb{R} P^{2}$, we must conclude that this map is precisely the Berry-Robbins wave function: $|\bar{\varphi}(\boldsymbol{r}) \equiv| \Psi(\boldsymbol{r})\rangle$. The consequences of this fact for the single-valuedness condition (27) are of the utmost importance since, as shown in [27] by means of an explicit example, it might well happen that $|\Psi(-\boldsymbol{r})\rangle=-|\Psi(\boldsymbol{r})\rangle$.

Therefore, although the purpose of the single-valuedness condition of Berry and Robbins is to incorporate indistinguishability already at the level of configuration space, the specific requirement (27) carries with itself certain ambiguities. This has been analyzed in full detail in [25] and [27]. Instead we propose the imposition of invariance on the wave function, in the sense of theorem 2.10, as a more clear and concise way to incorporate indistinguishability into the formalism of quantum mechanics.

$\S$ The configuration space is actually of the form $\mathbb{R}^{3} \times \mathbb{R}_{+} \times \mathbb{R} P^{2}$, but we are ignoring the first two factors, corresponding to the center of mass position vector and the relative distance between the particles, because they do not play any role in the discussion. 


\section{Conclusions and outlook}

In the present paper I have presented a mathematical framework for the study of quantum mechanics on spaces with a finite fundamental group which is based on the Gelfand-Naimark and Serre-Swan equivalences. The original motivation for this work has been the problem of quantum indistinguishability. An important aspect of this problem has been discussed using the tools developed in this paper.

Let us finish this paper with a brief outline of some problems for which the results presented in section 2 might be useful:

- Following the spirit of the Berry-Robbins approach, Peshkin has argued in [23] that spin zero particles must be bosons. Although his work has been criticized by Allen and Mondragon in [29], these authors do not provide a conclusive argument against the possibility of a topological origin of the spin-statistics connection. In his reply [62] and subsequent work [26], Peshkin still argues that, when taking indistinguishability into account, spin zero fermions can be disregarded. It would be interesting to spell out in detail Peshkin's calculations using the language presented here. This could provide a conclusive answer to that controversy.

- As remarked in our previous papers [51, 52, Kuckert's work [61] suggests a close connection between the spin-statistics connection, on one hand, and the angular momentum algebra for one and two particles and their possible intertwining relations, on the other. The techniques presented in this paper could be used to recast Kuckert's proposal in global language. A first step in this direction has been taken in [51], but a detailed analysis of the representation theoretic aspects of the problem remains to be done.

- Recently, new applications of geometric phases and topological invariants have appeared in the context of spin systems and in relation to quantum phase transitions. In particular, it has been shown in 63. that it is possible to relate certain topological invariants computed from the Hamiltonian and relate them to quantum criticality. The use of projective modules, as presented in the present paper, can be very convenient in this context from the computational point of view.

\section{Acknowledgments}

It is a pleasure to thank M. Paschke for his key guidance and encouragement during the initial phase of this work, and also to N.A. Papadopoulos and F. Scheck for the many enlightening discussions we had over a period of several years about this work. My intellectual debt to them will remain unmatched. Financial support from DAAD, Universidad de los Andes and Colciencias is gratefully acknowledged. 


\section{Appendix A. Projective modules and the Serre-Swan theorem}

Definition A.1. Let $R$ be a unital ring and $\mathcal{E}$ an abelian group. $\mathcal{E}$ is said to be a (left) $R$-module if there is a map $R \times \mathcal{E} \rightarrow \mathcal{E},(r, \varphi) \mapsto r \cdot \varphi$ such that, for all $r_{1}, r_{2} \in R$ and $\varphi, \varphi_{1}, \varphi_{2} \in \mathcal{E}$, the following relations hold:

$$
\begin{aligned}
& r \cdot\left(\varphi_{1}+\varphi_{2}\right)=r \cdot \varphi_{1}+r \cdot \varphi_{2} \\
& \left(r_{1}+r_{2}\right) \cdot \varphi=r_{1} \cdot \varphi+r_{2} \cdot \varphi \\
& \left(r_{1} r_{2}\right) \cdot \varphi=r_{1} \cdot\left(r_{2} \cdot \varphi\right) \\
& 1 \cdot \varphi=\varphi .
\end{aligned}
$$

Definition A.2. Let $\mathcal{E}$ be an $R$-module. A subset $S$ of $\mathcal{E}$ is said to be a set of generators for $\mathcal{E}$, if every $\varphi \in \mathcal{E}$ can be written as a sum $\varphi=\sum_{\sigma \in S} r_{\sigma} \cdot \sigma$, where $r_{\sigma} \in R$ and $r_{\sigma}=0$ for all but a finite number of elements $\sigma \in S$. If, in addition, the set $S$ is finite, we say that $\mathcal{E}$ is finitely generated. If $S$ is a set of generators for $\mathcal{E}$ and if for every $\varphi \in \mathcal{E}$ the expansion $\varphi=\sum_{\sigma \in S} r_{\sigma} \cdot \sigma$ is unique, we call $S$ a basis. A module $\mathcal{E}$ is called free if it admits a basis.

Given two $R$-modules $\mathcal{E}$ and $\mathcal{F}$, we can construct their direct sum $\mathcal{E} \oplus \mathcal{F}$ as the set of pairs $(\eta, \varphi)$ with $\eta \in \mathcal{E}$ and $\varphi \in \mathcal{F}$. Addition is defined componentwise, $(\eta, \varphi)+\left(\eta^{\prime}, \varphi^{\prime}\right)=\left(\eta+\eta^{\prime}, \varphi+\varphi^{\prime}\right)$ and the $R$-operation given by $r \cdot(\eta, \varphi)=(r \cdot \eta, r \cdot \varphi)$. Note that a ring $R$ can also be considered as a module over itself. We can therefore construct the $n$-fold sum $R^{n}=R \oplus \cdots \oplus R$, seen as a left $R$-module, with $R$-product $r \cdot\left(r_{1}, \ldots, r_{n}\right)=\left(r r_{1}, \ldots, r r_{n}\right)$. This module is free, with a standard basis given by the $n$ elements of the form $(0, \ldots, 0,1,0, \ldots, 0)$.

Proposition A.3 (cf.64, Proposition 4.1). Let $\mathcal{E}$ be a finitely generated free $R$-module, with basis $S=\left\{\sigma_{1}, \ldots, \sigma_{n}\right\}$. Then $\mathcal{E}$ is isomorphic to the $R$-module $R^{n}$.

Free modules are similar to vector spaces in the sense that if $S$ is a basis for $\mathcal{E}$, then $\sum_{\sigma \in S} r_{\sigma} \cdot \sigma=0$ implies $r_{\sigma}=0$ for all $\sigma \in S$. But, of course, there are modules which are not free.

Example A.4. Let $\mathbb{Z}_{n}$ denote the additive group of integers modulo $n$. Its elements are equivalence classes $[m]_{n}$, with $[m]_{n}=\left[m^{\prime}\right]_{n}$ if and only if $m=m^{\prime}(\bmod n)$ and addition defined by $[r]_{n}+[s]_{n}:=[r+s]_{n} . \mathbb{Z}_{n}$ can also be regarded as a ring, if we define the product by $[r]_{n}[s]_{n}:=[r s]_{n}$. Regarded as a module over itself, $\mathbb{Z}_{n}$ is a free module, generated by $[1]_{n}$. But we can also consider, say, $\mathbb{Z}_{2}$ as a $\mathbb{Z}_{6}$-module, by defining the ring operation through $[m]_{6} \cdot[n]_{2}:=[m n]_{2}$. In that case, one easily checks that $\mathbb{Z}_{2}$ is generated (over $\mathbb{Z}_{6}$ ) by $[1]_{2}$. But the set $S=\left\{[1]_{2}\right\}$ is not a basis, because $[1]_{2}$ satisfies certain relations as, for example, $[3]_{6} \cdot[1]_{2}=[1]_{2}$. This shows that $S$ is not linearly independent or, in other words, that $\mathbb{Z}_{2}$ is not a free $\mathbb{Z}_{6}$-module. Analogous computations show that $\mathbb{Z}_{3}$ is also a $\mathbb{Z}_{6}$-module which, again, is not free.

The following definition of projective module is not the most "elegant" one, but is well-suited for our purposes. 
Definition A.5. An $R$-module $\mathcal{P}$ is said to be projective if it is a direct summand of a free module.

Proposition A.6 (cf.[56], Proposition 2.22). An $R$-module $\mathcal{P}$ is projective if and only if it is of the form $p(\mathcal{F})$, where $\mathcal{F}$ is a free $R$-module and $p: \mathcal{F} \rightarrow \mathcal{F}$ an $R$-module homomorphism that is an idempotent, that is, such that $p^{2}=p$.

Example A.7. From the previous example we know that both $\mathbb{Z}_{2}$ and $\mathbb{Z}_{3}$ can be regarded as $\mathbb{Z}_{6}$-modules. It follows that $\mathbb{Z}_{2} \oplus \mathbb{Z}_{3}$ is also a $\mathbb{Z}_{6}$-module. In fact, it is isomorphic to $\mathbb{Z}_{6}$. The isomorphism is given by the following map:

$$
\begin{aligned}
\mathbb{Z}_{2} \oplus \mathbb{Z}_{3} & \longrightarrow \mathbb{Z}_{6} \\
\left([m]_{2},[n]_{3}\right) & \longmapsto[3 m+2 n]_{6} .
\end{aligned}
$$

Let us consider the $\mathbb{Z}_{6}$-linear map $p: \mathbb{Z}_{6} \rightarrow \mathbb{Z}_{6}$ defined by $p\left([m]_{6}\right):=[3 m]_{6}$. We have $p^{2}\left([m]_{6}\right)=[9 m]_{6}=[3 m+6 m]_{6}=p\left([m]_{6}\right)$, so that $p\left(\mathbb{Z}_{6}\right)$ is a projective module, given precisely by the image of $\mathbb{Z}_{2}$ under (A.2). We thus have $\mathbb{Z}_{2} \cong p\left(\mathbb{Z}_{6}\right)$, illustrating the previous proposition.

Example A.8. Let $M$ be a compact manifold and $E \stackrel{\pi}{\rightarrow} M$ a (real/complex) vector bundle. Then the space $\Gamma(E)$ of all continuous sections of the bundle has the structure of a finitely generated projective module over the algebra $C(M)$ of continuous (real/complex) functions on $M$. In fact, the space $C(M)$, being an algebra, is also a ring, with respect to pointwise addition and multiplication of functions. $\Gamma(E)$ is a vector space over $\mathbb{K}(=\mathbb{R}, \mathbb{C})$ and, in particular, an abelian group. The ring operation is given by the product $C(M) \times \Gamma(E) \rightarrow \Gamma(E),(f, \sigma) \mapsto f \cdot \sigma$, pointwise defined: $(f \cdot \sigma)(x):=f(x) \sigma(x)$. The fact that this module is finitely generated and projective is part of the Serre-Swan theorem [65, 66]. This theorem states that there is an equivalence between the categories of vector bundles over compact spaces $M$, on one side, and of finitely generated projective modules over the respective algebras $C(M)$, on the other.

Remark A.9. A detailed proof of this theorem can be found, e.g., in [56]. For the purpose of this paper, the following information about the construction of the equivalence will be sufficient:

- A vector bundle with typical fibre $V$ can be completely described by an open cover $\left\{U_{1}, \ldots, U_{m}\right\}$ of $M$, together with a family of transition functions $g_{i j}: U_{i} \cap U_{j} \rightarrow$ $\mathrm{Gl}(V)$ satisfying certain compatibility (cocycle) conditions [67]. Let $\left\{\psi_{j}\right\}_{j}$ be a partition of unity subordinate to the cover $\left\{U_{j}\right\}_{j}$ (here we use the convention $\left.\sum_{j} \psi_{j}^{2}=1\right)$. Set $N=m \operatorname{dim}(V)$. Then, the projective module corresponding to this bundle is defined by an idempotent $P: C(M)^{N} \rightarrow C(M)^{N}$, acting as an $N \times N$ matrix-valued function constructed from $\operatorname{dim}(V)^{2}$ blocks, the $i j$-block of which is given by $g_{i j} \psi_{i} \psi_{j}$.

- On the other hand, it follows from proposition A.6 that any finitely generated projective module over $C(M)$ is given by a $C(M)$-valued matrix idempotent $P$ as $P\left(C(M)^{N}\right)$, for some $N$. In this case, the fibre of the corresponding vector bundle 
over the point $x \in M$ is given by $P(x)\left(\mathbb{K}^{N}\right)$, where $\mathbb{K}=\mathbb{R}, \mathbb{C}$, depending on whether we are working with real, respectively complex functions.

- A vector bundle of rank $r$ which is not trivial will give place to a projective module which is not free. Thus, finding a set of $r$ linearly independent, nowhere-vanishing sections of such a bundle amounts, at the algebraic level, to find a basis for the module.

Example A.10. The Möbius bundle is the simplest example of a nontrivial real line bundle. Using the coordinate $\theta$ to denote points in the circle $S^{1}(0 \leq \theta \leq 2 \pi)$, we can cover it with two open neighbourhoods, the first one, $U_{a}$, including all points of $S^{1}$ but the one corresponding to $\theta=0$ and the second one, $U_{b}$, including all points of $S^{1}$ but the one corresponding to $\theta=\pi$. Using the bundle construction theorem, we can construct a real line bundle by specifying transition functions $g_{a b}: U_{a} \cap U_{b} \rightarrow \operatorname{Gl}(n, \mathbb{R})$ satisfying the usual cocycle conditions (in this case $n=1$ ). Since $U_{a} \cap U_{b}$ has two connected components, we can define

$$
g_{a b}(\theta):= \begin{cases}+1 & \text { for } 0<\theta<\pi \\ -1 & \text { for } \pi<\theta<2 \pi\end{cases}
$$

Putting $g_{b a}=g_{a b}$ and $g_{a a}=g_{b b}=1$, we obtain transition functions that are locally constant (hence continuous) and that give place to a real line bundle with the topology of an open Möbius strip. Recall now that every vector bundle can be expressed as a subbundle of a trivial bundle of higher rank. In the case of the Möbius bundle, this allows us to visualize the bundle in three dimensions. Consider the trivial bundle over $S^{1}$ with total space $S^{1} \times \mathbb{R}^{3}$. The Möbius bundle can be described as the subbundle of this trivial bundle whose fibre over $\theta$ is the real line generated by the vector $v(\theta)=(0, \sin (\theta / 2), \cos (\theta / 2))$. Note that, as we go around the circle, this vector rotates through an angle of $\pi$, thus generating the twist of a Möbius strip. The description of this bundle by means of a projector is as follows. For each $\theta$ we can consider the projection from $\mathbb{R}^{3}$ onto the line generated by $v(\theta)$. Since the first component of $v(\theta)$ is always zero, we may consider only the projection from the $y$ - $z$ plane. A simple calculation shows that the matrix form of this projector is

$$
P(\theta)=\frac{1}{2}\left(\begin{array}{cc}
1-\cos \theta & \sin \theta \\
\sin \theta & 1+\cos \theta
\end{array}\right) .
$$

Let $\mathcal{R}:=C\left(S^{1}, \mathbb{R}\right)$ denote the algebra of continuos, real functions on the circle. Notice that, although the components of $v$ do not belong to $\mathcal{R}$, the components of $P$ certainly do. The projective $\mathcal{R}$-module associated to the bundle is thus given by $P\left(\mathcal{R}^{2}\right)$. Explicitly, the elements of the module are vector valued maps $\sigma: S^{1} \mapsto \mathbb{R}^{2}$ of the form $\sigma=a \sigma_{1}+b \sigma_{2}$, where $a, b \in \mathcal{R}$ and $\sigma_{1}$ and $\sigma_{2}$ are the two columns of $P$. This is precisely the space of sections of the bundle the total space of which is given (as a set) by $\left\{(\theta, \lambda v(\theta)) \in S^{1} \times \mathbb{R}^{3} \mid \lambda \in \mathbb{R}\right\}$. Notice also that if we define $\psi_{a}(\theta):=|\sin (\theta / 2)|$ for $\theta \neq 0,2 \pi$, with $\psi_{a}(0) \equiv \psi_{a}(2 \pi):=0$ and, similarly, $\psi_{b}(\theta):=|\cos (\theta / 2)|$ for $\theta \neq \pi$, with $\psi_{b}(\pi):=0$, then $\operatorname{supp}\left(\psi_{i}\right) \subseteq U_{i}$ and $\psi_{i} \in \mathcal{R}(i=a, b)$. One then checks that the 
components of $P$ are precisely given by the functions $g_{i j} \psi_{i} \psi_{j}$ (with $i, j=a, b$ and $g_{i j}$ as in (A.3) $)$, in accordance with remark A.9.

\section{Appendix B. The regular representation and projection operators}

Let $G$ be a finite group of order $|G|$. Denote with $\rho_{i}: G \rightarrow \operatorname{Gl}\left(U^{i}\right)$ the inequivalent irreducible representations of $G$, of dimension $n_{i}=\operatorname{dim} U^{i}, i=1, \ldots, N$. If $\mathcal{F}(G)$ denotes the vector space of complex functions on $G$, then the regular representation of $G$ is the one on $\mathcal{F}(G)$ which is induced by the group multiplication. It is a well-known fact that the regular representation contains all irreducible representations of $G$, each one with a multiplicity equal to its dimension [69]:

$$
\mathcal{F}(G) \cong \bigoplus_{i=1}^{N} n_{i} U^{i}
$$

Consider now $\mathcal{F}(G)$ as a representation space for $G \times G$, where the representation $\hat{r}^{G}$ of $G \times G$ on $\mathcal{F}(G)$ is induced by the action

$$
\begin{aligned}
& (G \times G) \times G \longrightarrow G \\
& \left(\left(g_{1}, g_{2}\right), h\right) \longmapsto g_{1} h g_{2}^{-1} .
\end{aligned}
$$

There is a close relation between the direct sum in (B.1) and the decomposition of $\hat{r}^{G}$ into irreducible $G \times G$ representations. It is obtained in the following way. The representation $\rho_{i}$ induces a representation $\tilde{\rho}_{i}$ of $G$ on the dual space $U^{i *}$ (which is also irreducible and is defined through $\left(\tilde{\rho}_{i}(g) \varphi\right)(u):=\varphi\left(\rho_{i}\left(g^{-1}\right) u\right)$, with $\varphi \in U^{i *}$ and $\left.u \in U^{i}\right)$ and it turns out that, as a $G \times G$ representation, $\rho_{i} \otimes \tilde{\rho}_{i}$ is irreducible. Explicitly, we have:

$$
\begin{aligned}
\rho_{i} \otimes \tilde{\rho}_{i}: G \times G & \longrightarrow \operatorname{Gl}\left(U^{i} \otimes U^{i *}\right) \\
(a, b) & \longmapsto \rho_{i}(a) \otimes \tilde{\rho}_{i}(b) .
\end{aligned}
$$

Irreducibility follows directly from the fact that $\operatorname{tr}\left(\rho_{i}(a) \otimes \tilde{\rho}_{i}(b)\right)=\operatorname{tr} \rho_{i}(a) \operatorname{tr} \tilde{\rho}_{i}(b)$. Consider now, for each $i$, the linear map $S_{i}: U^{i} \otimes U^{i *} \rightarrow \mathcal{F}(G)$, defined on simple tensors by the formula $S_{i}(u \otimes \varphi)(g):=\varphi\left(\rho_{i}\left(g^{-1}\right) u\right)$, where $g \in G, u \in U^{i}$ and $\varphi \in U^{i *}$. It is easy to see, using the orthogonality relations for the matrix elements of $\rho_{i}$, that the map $S_{i}$ is injective. Moreover, it is $G \times G$-equivariant. This follows directly from the definitions given above since, for $(a, b) \in G \times G, u \otimes \varphi \in U^{i} \otimes U^{i *}$ and $g \in G$, we have:

$$
\begin{aligned}
S_{i}((a, b) \cdot u \otimes \varphi)(g) & \equiv S_{i}\left(\rho_{i}(a) u \otimes \tilde{\rho}_{i}(b) \varphi\right)(g) \\
& =\left(\tilde{\rho}_{i}(b) \varphi\right)\left(\rho_{i}\left(g^{-1}\right) \rho_{i}(a) u\right) \\
& =\varphi\left(\rho_{i}\left(\left(a^{-1} g b\right)^{-1}\right) u\right) \\
& =S_{i}(u \otimes \varphi)\left((a, b)^{-1} \cdot g\right) \\
& =\left(\hat{r}^{G}(a, b) S_{i}(u \otimes \varphi)\right)(g) \\
& \equiv\left((a, b) \cdot S_{i}(u \otimes \varphi)\right)(g) .
\end{aligned}
$$


From this and the injectivity of $S_{i}$ it follows, from Schur's lemma, that the representations $\left(U^{i} \otimes U^{i *}, \rho_{i} \otimes \tilde{\rho}_{i}\right)$ and $\left(\left.\mathcal{F}(G)\right|_{\operatorname{Im}\left(S_{i}\right)},\left.\hat{r}^{G}\right|_{\operatorname{Im}\left(S_{i}\right)}\right)$ must be equivalent. But then, taking the sum of all $S_{i}$, we obtain a bijective linear map

$$
S: \bigoplus_{i=1}^{N}\left(U^{i} \otimes U^{i *}\right) \longrightarrow \mathcal{F}(G)
$$

that furnishes an equivalence between $\left(\bigoplus_{i=1}^{N}\left(U^{i} \otimes U^{i *}\right), \bigoplus_{i=1}^{N}\left(\rho_{i} \otimes \tilde{\rho}_{i}\right)\right)$ and $\left(\mathcal{F}(G), \hat{r}^{G}\right)$.

The isomorphism $S$ can be used to obtain an explicit formula for the projection from $\mathcal{F}(G)$ to the copy of $U^{i} \otimes U^{i *}$ inside $\mathcal{F}(G)$, as explained below. Chose a basis $\left\{e_{r}^{(i)}\right\}_{r}$ for $U^{i}$ and let $\left\{\tilde{e}_{r}^{(i)}\right\}_{r}$ be the dual basis of $U^{i *}$, induced by $\left\{e_{r}^{(i)}\right\}_{r}$. Denote with $R^{(i)}(g)$ the representing matrices of $\rho_{i}$, with respect to $\left\{e_{r}^{(i)}\right\}_{r}$ :

$$
\rho_{i}(g)\left(e_{r}^{(i)}\right)=\sum_{r^{\prime}=1}^{n_{i}} R_{r^{\prime}, r}^{(i)}(g) e_{r^{\prime}}^{(i)}
$$

Now, every function $f \in \mathcal{F}(G)$ can be written in the form $f=\sum_{g \in G} f(g) \delta_{g}$, where $\delta_{g}$ is the characteristic function

$$
\delta_{g}(h):= \begin{cases}1 & \text { if } h=g, \\ 0 & \text { if } h \neq g .\end{cases}
$$

To obtain the projection operators, it is sufficient to compute $S^{-1}\left(\delta_{g}\right)$. We therefore seek the (unique, $g$-dependent) coefficients $\lambda_{r, r^{\prime}}^{i}$ such that

$$
\delta_{g}=S\left(\sum_{i=1}^{N} \sum_{r, r^{\prime}=1}^{n_{i}} \lambda_{r, r^{\prime}}^{i} e_{r}^{(i)} \otimes \tilde{e}_{r^{\prime}}^{(i)}\right) .
$$

Using the definition of $S$, we obtain:

$$
\begin{aligned}
\delta_{g}(h) & =S\left(\sum_{i=1}^{N} \sum_{r, r^{\prime}=1}^{n_{i}} \lambda_{r, r^{\prime}}^{i} e_{r}^{(i)} \otimes \tilde{e}_{r^{\prime}}^{(i)}\right)(h) \\
& =\sum_{i=1}^{N} \sum_{r, r^{\prime}=1}^{n_{i}} \lambda_{r, r^{\prime}}^{i} S_{i}\left(e_{r}^{(i)} \otimes \tilde{e}_{r^{\prime}}^{(i)}\right)(h)=\sum_{i=1}^{N} \sum_{r, r^{\prime}=1}^{n_{i}} \lambda_{r, r^{\prime}}^{i} \tilde{e}_{r^{\prime}}^{(i)}\left(\rho_{i}\left(h^{-1}\right) e_{r}^{(i)}\right) \\
& =\sum_{i=1}^{N} \sum_{r, r^{\prime}, r^{\prime \prime}=1}^{n_{i}} \lambda_{r, r^{\prime}}^{i} R_{r^{\prime \prime}, r}^{(i)}\left(h^{-1}\right) \tilde{e}_{r^{\prime}}^{(i)}\left(e_{r^{\prime \prime}}^{(i)}\right)=\sum_{i=1}^{N} \sum_{r, r^{\prime}=1}^{n_{i}} \lambda_{r, r^{\prime}}^{i} R_{r^{\prime}, r}^{(i)}\left(h^{-1}\right) .
\end{aligned}
$$

Multiplying the last equation by $R_{k, l}^{(j)}(h)$ and summing over $h \in G$ we then obtain, using the orthogonality relations:

$$
\lambda_{r, r^{\prime}}^{i}=\frac{n_{i}}{|G|} R_{r, r^{\prime}}^{(i)}(g) .
$$

This implies, for $f \in \mathcal{F}(G)$,

$$
f(g)=\sum_{h \in G} f(h) \delta_{h}(g)=\sum_{i=1}^{N} \sum_{r=1}^{n_{i}} \frac{n_{i}}{|G|} \sum_{h \in G} R_{r, r}^{(i)}\left(h^{-1}\right) f\left(h^{-1} g\right) .
$$


This shows that the operator $\frac{n_{i}}{|G|} \sum_{r=1}^{n_{i}} \sum_{h \in G} R_{r, r}^{(i)}\left(h^{-1}\right) h \cdot(-)$ is the projection operator $\mathcal{F}(G) \rightarrow U^{i} \otimes U^{i *}$. Moreover, since $U^{i} \otimes U^{i *}$ is isomorphic to the $n_{i}$-fold sum $U^{i} \oplus \cdots \oplus U^{i}$, it is natural to consider the operators

$$
P_{r, s}^{(i)}:=\frac{n_{i}}{|G|} \sum_{h \in G} R_{r, s}^{(i)}\left(h^{-1}\right) h \cdot(-) .
$$

It turns out that these operators allow one to explicitly obtain the $r^{\text {th }}$ copy of $U^{i}$ inside $\mathcal{F}(G)$. Equation (B.12) is the starting point for the construction of a decomposition of the algebra $C(M)$ into a direct sum of projective modules. The reader might have noticed that these operators are also used in the field of Quantum Chemistry, e.g. for the construction of symmetry-adapted basis (cf. [70]).

\section{References}

[1] Fierz M 1939 On the relativistic theory of force free particles of arbitrary spin Helv. Phys. Acta $123-37$

[2] Pauli W 1940 The connection between spin and statistics Phys. Rev. 58 716-22

[3] Duck I and Sudarshan E C G 1997 Pauli and the Spin-Statistics Theorem (Singapore: World Scientific)

[4] Streater R and Wightman A 2000 Spin, Statistics and all That (Princeton: Princeton University Press)

[5] Haag R 1996 Local Quantum Physics (Texts and Monographs in Physics) (Berlin: Springer Verlag, 2nd edition)

[6] Sudarshan E C G 1975 Relation between spin and statistics Stat. Phys. Suppl.: J. Indian Inst. Sci. June 123-37

[7] Balachandran A P, Daughton A, Gu Z C, Marmo G, Sorkin R D and Srivastava A M 1990 A topological spin-statistics theorem or a use of the antiparticle Mod. Phys. Lett. A 5 1575-85

[8] Finkelstein D and Rubinstein J 1968 Connection between spin, statistics, and kinks J. Math. Phys. 9 1762-79

[9] Tscheuschner R D 1989 Topological spin-statistics relation in quantum field theory Int. J. Theor. Phys. 28 1269-1310

[10] Mickelsson J 1984 Geometry of spin and statistics in classical and quantum mechanics Phys. Rev. D 30 1843-1845, 1984.

[11] Berry M V and Robbins J M 1997 Indistinguishability for quantum particles: spin, statistics and the geometric phase Proc. R. Soc. London A 453 1771-90

[12] M.V. Berry and J.M. Robbins 2000 Quantum indistinguishability: alternative constructions of the transported basis J. Phys. A: Math. Theor. 33 L207-14

[13] Berry M V and Robbins J M 2000 Spin-Statistics Connection and Commutation Relations: Experimental Tests and Theoretical Implications (AIP Conference Proceedings vol 545) ed R C Hilborn and G M Tino (American Institute of Physics) pp 3-15

[14] Berry M V 2008 Three quantum obsessions Nonlinearity 21 T19-26

[15] Twamley J 1997 Statistics given a spin Nature $\mathbf{3 8 9} 127$

[16] Sudarshan E C G and Duck I M 2003 What price the spin-statistics theorem? Pramana Journal of Physics 61 1-9

[17] Chruściński D and Jamiołkowski A 2004 Geometric Phases in Classical and Quantum Mechanics (Boston: Birkhuser)

[18] Wightman A 2000 The spin-statistics connection: Some pedagogical remarks in response to Neuenschwander's question Electron. J. Diff. Eqns. Conf.04 207-13

[19] Atiyah M 2001 Configurations of points Phil. Trans. Roy. Soc. Lond. A 359 1375-87 
[20] Atiyah M and Sutcliffe P 2002 The geometry of point particles Proc. R. Soc. London A 458 $1089-115$

[21] Atiyah M and Bielawski R 2002 Nahm's equations, configurations spaces and flag manifolds Bull. Brazilian Math. Soc. 33 157-66

[22] Anandan J 1998 Spin-statistics connection and relativistic Kaluza-Klein space-time Phys. Lett. A 248 124-30

[23] Peshkin M 2003 Spin and statistic in nonrelativistic quantum mechanics: The spin-zero case Phys. Rev. A 67042102

[24] Harrison J M and Robbins J M 2004 Quantum indistinguishability from general representations of $\mathrm{su}(2 \mathrm{n})$ J. Math. Phys. 45 1332-58

[25] Papadopoulos N A, Paschke M, Reyes A and Scheck F 2004 The spin-statistics relation in nonrelativistic quantum mechanics and projective modules Annales Mathématiques BlaisePascal 11 205-20

[26] Peshkin M 2006 Spin-zero particles must be bosons: A new proof within nonrelativistic quantum mechanics Found. Phys. 36 19-29

[27] Papadopoulos N A and Reyes-Lega A F 2010 On the geometry of the Berry-Robbins approach to spin-statistics Found. Phys. 4082951

[28] Paschke M 2001 Von Nichtkommutativen Geometrien, ihren Symmetrien und etwas Hochenergiephysik Dissertation Johannes Gutenberg University, Mainz (MZ-TH/01-T2)

[29] Allen R and Mondragon A 2003 Comment on "Spin and statistics in nonrelativistic quantum mechanics: The spin-zero case" Phys. Rev. A 68046101

[30] Neuenschwander D E 1994 Question no. 7. The spin-statistics theorem Am. J. Phys. 62972

[31] Hilborn R C 1995 Answer to question no. 7 ["The spin-statistics theorem," Dwight E. Neuenschwander, Am. J. Phys. 62 (11), 972 (1994)] Am. J. Phys. 63298

[32] Romer R H 2002 The spin-statistics theorem Am. J. Phys. 70791

[33] Duck I and Sudarshan E C G 1998 Toward an understanding of the spin-statistics theorem Am. J. Phys. 66 284-303

[34] Isham C J 1984 Relativity, Groups and Topology II (Les Houches, 1983) ed B S DeWitt and R Stora (Amsterdam: Kluwer Academic Press) pp 1059-290

[35] Pauli W 1939 Über ein Kriterium für ein- oder Zweiwertigkeit der Eigenfunktionen in der Wellenmechanik Helv. Phys. Acta 12 147-68

[36] Pauli W 1958 Enc. Phys. 545

[37] Bopp F and Haag R 1950 Über die Möglichkeit von Spinmodellen Z. Naturforsch 5a 644-53

[38] Schulman L 1968 A path integral for spin Phys. Rev. 5 1558-69

[39] Laidlaw M and DeWitt-Morette C 1971 Feynman functional integrals for systems of indistinguishable particles Phys. Rev. D 3 1375-78

[40] Souriau J M 1969 Structure des Systèmes Dynamiques (Paris: Dunod)

[41] Dowker J S 1972 Quantum mechanics and field theory on multiply connected and on homogeneous spaces J. Phys. A: Gen. Phys 5936

[42] Leinaas J M and Myrheim J 1977 On the theory of identical particles Il Nuovo Cimento 37B 1-23

[43] Woodhouse N 1980 Geometric Quantization (Oxford: Clarendon Press)

[44] Horvathy P A, Morandi G and Sudarshan E C G 1989 Inequivalent quantizations in multiply connected spaces Il Nuovo Cimento 11D 201-28

[45] Morandi G 1992 The Role of Topology in Classical and Quantum Physics (Lecture Notes in Physics vol m-7) (Berlin: Springer-Verlag)

[46] Boya L J and Sudarshan E C G 2007 The spin-statistics theorem in arbitrary dimensions Int. J. Theor. Phys. 46 3285-93

[47] Dowker H F and Sorkin R D 1998 A spin-statistics theorem for certain topological geons Class. Quant. Grav. 15 1153-67

[48] Alexanian G and Balachandran A P 2002 On the failure of spin-statistics connection in quantum gravity Phys.Lett. B 537 103-16 
[49] Balachandran A P, Mangano G, Pinzul A, and Vaidya S 2006 Spin and statistics on the Groenewold-Moyal plane: Pauli-forbidden levels and transitions Int. J. Mod. Phys. A 21 3111-26

[50] Milotti E, Curceanu (Petrascu) C, Adler S L and Berry M V 2010 Special issue on spin statistics. Found. Phys. 40 681-83

[51] Reyes-Lega A F and Benavides C 2010 Remarks on the configuration space approach to spinstatistics Found. Phys. 40 1004-29

[52] Benavides C and Reyes-Lega A F 2010 Geometric and Topological Methods for Quantum Field Theory (Villa de Leyva, 2007) ed H Ocampo et al (Cambridge: Cambridge University Press) pp $344-67$

[53] Simon B 1996 Representations of Finite and Compact Groups (Graduate Studies in Mathematics vol 10) (Providence: American Mathematical Society)

[54] Boothby W M 2002 An Introduction to Differentiable Manifolds and Riemannian Geometry (New York: Academic Press)

[55] Milnor J 1957 Commun. Math. Helv. 32215

[56] Gracia-Bondía J M, Várilly J C and Figueroa H 2001 Elements of Noncommutative Geometry (Boston: Birkhäuser)

[57] Atiyah M F 1967 K-theory (New York: Benjamin)

[58] Madsen I and Tornehave J 1997 From Calculus to Cohomology: De Rham cohomology and characteristic classes (Cambridge: Cambridge University Press)

[59] Kastrup H A 2006 Quantization of the canonically conjugate pair angle and orbital angular momentum Phys. Rev. A 73052104

[60] Rehacek J, Bouchal Z, Celechovsky R, Hradil Z and Snchez-Soto L L 2008 Experimental test of uncertainty relations for quantum mechanics on a circle Phys. Rev. A 77032110

[61] Kuckert B 2004 Spin and statistics in nonrelativistic quantum mechanics, I Phys. Lett. A 322 $47-53$

[62] Peshkin M 2003 Reply to comment on "Spin and statistics in nonrelativistic quantum mechanics: The spin-zero case" Phys. Rev. A 68046102

[63] Contreras H and Reyes-Lega A F 2008 Berry phases, quantum phase transitions and Chern numbers Physica B 403 1301-2

[64] Hilton P J and Stammbach U 1971 A Course in Homological Algebra (Berlin: Springer Verlag)

[65] Serre J P 1957 Modules projectifs et espaces fibrés à fibre vectorielle Séminaire Dubreil 2358

[66] Swan R G 1962 Vector bundles and projective modules Trans. Amer. Math. Soc. 105 264-77

[67] Nakahara M 1990 Geometry, Topology and Physics (Graduate Student Series in Physics) (Bristol: Institute of Physics Publishing)

[68] Mackey G W Unitary Group Representations in Physics, Probability, and Number Theory (Reading, MA: Benjamin-Cummings)

[69] Sternberg S 1994 Group Theory and Physics (Cambridge: Cambridge University Press)

[70] Atkins P W and Friedman R S 1999 Molecular Quantum Mechanics (Oxford: Oxford University Press, 3 ed.) 